\title{
Optimising 3D scaffold for otic neural progenitor differentiation
}

\author{
Kamolchanok Ngamkham ${ }^{1,2}$, Marcelo N. Rivolta ${ }^{2}$, Giuseppe Battaglia ${ }^{1}$ \\ ${ }^{1}$ Department of Chemistry, University College London, London, WC1H OAJ, \\ UK. \\ ${ }^{2}$ Department of Biomedical Sciences, University of Sheffield, Sheffield, South \\ Yorkshire, S10 2TN, UK.
}

Correspondence to: Giuseppe Battaglia. Department of Chemistry, University College London, London, WC1H OAJ, UK. Telephone: +44(0)2076794688; email: g.battaglia@ucl.ac.uk. 


\section{ABSTRACT}

Hearing loss is a condition highly prevalent worldwide. It affects people

3 of a broad age range since the causes and risk factors are varied. At present,

4 some types of hearing impairments have a palliative treatment whereas some,

5 especially for those where otic neurons are damaged, cannot be properly

6 treated. Recent findings had shown it possible to use human embryonic stem

7 cell-derived otic neural progenitors (ONPs) as a new mode of treating hearing

8 loss caused by damage to the spiral ganglion neurons (SGNs). To improve

9 the efficiency and overcome some limitations of this potential treatment, we

10 have applied principles of tissue engineering which involves an interaction

11 between cells and an extracellular matrix -mimicking scaffold. Here, we

12 describe the influence of poly(I-lactic acid)(PLLA) aligned fibres on ONP cell

13 morphology, proliferation, neuronal differentiation and establishment of neural

14 polarity under both progenitor and neuralising conditions. The results show

15 that most of ONPs on aligned fibres exhibited bipolar morphology and

16 extended their neurites along the major fibre axis. Their proliferation was

17 lower than those in 2D culture but the differentiation of ONPs on aligned fibres

18 was significantly enhanced in both progenitor and neuralising conditions as

19 indicated by the fluorescence intensity and number of cells that were positive

20 for neuronal markers ( $\beta$-tubulin III and NF200) and the expression pattern of

21 spiral ganglion molecular markers (MMP13, NPR2 and NTNG1). Moreover,

22 axonal and dendritic markers (TAU and MAP2 respectively) were also

23 induced after 14 days in culture. 
25 KEYWORDS. Otic neural progenitors, stem cells, spiral ganglion neurons,

26 electrospinning, aligned fibres, differentiation

\section{INTRODUCTION}

Hearing loss is one of the most common causes of disability. According

30 to the available data from the World Health Organization (WHO), around 360

31 million people worldwide are suffering from moderate or greater hearing loss.

32 Several types of hearing loss can be categorised according to the area of the

33 ear where the problem lies. Sensorineural hearing loss, which is caused by

34 the damage to the hair cells or the auditory nerve in the inner ear is the most

35 common. Unlike other nonmammalian vertebrates, damage or degeneration

36 of human hair cells and spiral ganglion neurons (SGNs) is irreversible ${ }^{1,2}$.

37 Even though cochlear implants can be used to overcome a hair cell deficit,

38 loss of SGNs can limit the effectiveness of this treatment ${ }^{3}$. Thus, alternative

39 strategies for SGN regeneration have been widely researched and stem cell

40 therapy has been proposed as a potential therapeutic path.

Findings from a recent study highlight the possibility of SGNs restoration using a stem cell-based strategy. Otic neural progenitors (ONPs)

43 that were differentiated from human embryonic stem cells were found to

44 exhibit true otic characteristics as indicated by the expression of several otic 45 markers such as PAX8, PAX2, SOX2 and FOXG1. They were also able to

46 further differentiate into SGNs under 'neuralising' condition, becoming positive 47 for neuronal markers, displaying appropriated membrane currents and firing 
48

action potentials. When transplanted into gerbils that were deafened by the application of ouabain (a model of auditory neuropathy), they engrafted and significantly improved the auditory thresholds ${ }^{4}$.

However, there are some potential limitations in this study that might constrain a wider application of this approach. The deafness-inducing drug ouabain damages primarily the type I SGNs, while type II SGNs and glial cells remain mostly intact ${ }^{5}$. These preserved tracks might act as a guidance cue for the transplanted cells, facilitating them to reach their targets centrally and on the periphery. In cases were these tracts are absent, such as in a cochlear nerve aplasia, ONPs might not be able to extend their neurites effectively and functional restoration might be impaired. Moreover, there are some putative drawbacks in delivering cells directly by cell injection. Based on evidence from other experimental systems, the retention of cells at the target site and cell survival rate can be poor ${ }^{6,7}$. To overcome those limitations, the use of a support or tissue engineered scaffold mimicking the extracellular matrix may be advantageous.

The complexity of the cochlea and sound sensing mechanism make tissue engineering for the inner ear a difficult task ${ }^{8}$. Most of the promising previous work relies on the improvement of the cochlear implant biological interface. . Various reports indicated that alteration of electrode surface topography, chemistry or biological functionalisation offer a great opportunity for the improvement of this prostheses ${ }^{9-20}$. 
70 The physiological extracellular matrix of the inner ear has also been studied

71 as a natural, tissue engineered scaffold. Mice, rat and human cochlea were

72 decellularised and extracellular matrix contents, identified. However, these

73 decellularised matrices will not be appropriated for clinical application of

74 transplanted cells, and have not been widely used to support stem cell

75 differentiation ${ }^{21}$.In this study, we aimed to investigate the behaviour of ONPs

76 on a biomaterial and explore if this scaffold could provide advantages for

77 ONP transplantation. Poly(l-lactic acid)(PLLA), a biodegradable and

78 biocompatible polymer, was fabricated as aligned electrospun fibres and used

79 as a scaffold for ONP culture. To address the effect of aligned fibres on

80 ONPs, cell attachment, proliferation, differentiation and establishment of

81 neural polarity were then analysed.

MATERIALS AND METHODS

Human embryonic stem cell (hESC) culture

Shef1 hESC line was cultured and used for the derivation of otic neural

86 progenitor. hESC colonies were maintained on inactivated mouse embryonic

87 fibroblast (MEF) feeder layers on gelatin-coated tissue culture flasks. The

88 culture media consisted of knockout Dulbecco's modified Eagle's medium

89 (Invitrogen), 20\% knockout serum replacement (KOSR), 1\% nonessential

90 amino acids, $2 \mathrm{mM}$ L-glutamine, (all from Invitrogen), $0.1 \mathrm{mM}$ ß-

91 mercaptoethanol (Sigma), and $4 \mathrm{ng} / \mathrm{ml}$ of basic fibroblast growth factor 
92 (bFGF; R\&D systems). The colonies were passaged in clumps every 7 days

93 using collagenase and mechanical dissociating methods.

Differentiation of hES cells into otic neural progenitors (ONPs) and sensory neurons

Generation of otic neuroprogenitors was performed as described in Chen et al 2012. Briefly, undifferentiated hESCs were dissociated to single cells using $0.025 \%$ trypsin-EDTA (Sigma). After trypsinization, cell suspension was pelleted at $157 \mathrm{rcf}$ for $5 \mathrm{~min}$ and passed through a $100 \mu \mathrm{m}$ cell strainer (BD Labware) to get completely dissociated cells or very small aggregates.

101 Cells were counted and plated onto laminin-coated T12.5 flasks (R\&D systems, $5 \mu \mathrm{g} / \mathrm{cm}^{2}$ ) at the seeding density of 4000 cells $/ \mathrm{cm}^{2}$. Cells were 103 maintained in a 1:1 mixture of Dulbecco's modified Eagle's medium (DMEM) 104 and F12 nutrient supplemented with N2 and B27 (DFNB). FGF3 (50ng/ml) 105 and FGF10 $(50 \mathrm{ng} / \mathrm{ml})$ were added to the medium as supplements to drive the 106 differentiation ${ }^{4,22}$. The differentiated medium was replaced every 2 days. After 107 3-4 days, other epithelial-like and surrounding cells were scraped off to enrich 108 for ONPs. The flask was cleaned from time to time and ONPs were then 109 allowed to grow for further 10-12 days. The purified ONPs were then 110 maintained in otic stem cell full medium or OSCFM (DFNB supplemented with 111 bFGF (20ng/ml), IGF1 (50ng/ml) and EGF $(20 \mathrm{ng} / \mathrm{ml}))$ and expanded on $0.1 \%$ 112 gelatin-coated flask until sufficient cell numbers were obtained. 
$115 \mathrm{ng} / \mathrm{ml}$ each) added at different time points as previously described by Chen et al. 2009 and $2012^{4,22}$.

119 in 3:1 chloroform: methanol respectively. The solution was stirred for 1 hour at 120 room temperature or until the polymer was totally dissolved. Three $\mathrm{ml}$ of 121 polymer solution was electrospun from a $3 \mathrm{ml}$ plastic syringe that attached to 122 a flat tip needle at constant flow rate, which was controlled by a multispeed 123 syringe pump. A high voltage $(\sim 10-12 \mathrm{kv})$ was applied to the needle tip to 124 charge the polymer solution and form a liquid jet. An aluminum-rotating disk 125 was used as a grounded collector to collect the electrospun fibres. Random 126 and high alignment of fibres was collected at the rotation speed of 180 and 1272000 rpm respectively.

\section{Scanning electron microscopy (SEM)}

130 visualised by SEM (Philips XL-20) at an accelerating voltage of $20 \mathrm{kV}$. Each

131 scaffold was coated with gold using a sputter coater (Emscope SC 500) prior 132 to the observation. The images were then analysed using image $\mathrm{J}$ software $133(\mathrm{NIH})$. 

that were sterilized in cold $70 \%$ ethanol for 15 min, washed 3 times with cold PBS and incubated in OSCFM medium overnight. About 8000 ONPs were 138 seeded onto a $1 \mathrm{~cm}^{2}$ scaffold membrane, using a very small volume $(20 \mu \mathrm{l})$.

139 To allow attachment to the scaffold, cells were allowed to settle for one hour 140 before the medium was topped up. ONPs were then maintained in either 141 progenitor (OSCFM) or neuralising conditions for up to 14 days. Size of 142 scaffolds and cell density were scaled up for qRT-PCR gene expression 143 analysis $\left(5 \times 10^{5}\right.$ cells/ $4 \mathrm{~cm}^{2}$ scaffold) in order to obtain good RNA yields.

\section{SEM sample preparation for ONPs on 3D scaffolds}

145 As ONPs are loosely attached cells and most of them were not preserved 146 under normal preparation, a new preparation protocol was established 147 especially for ONPs. Culture medium was removed from the wells and the 148 cells were fixed with warm Karnovsky's fixative, which is a mixture of $2 \%$ 149 paraformaldehyde, 2.5\% glutaraldehyde and 0.1M Sodium Phosphate buffer, 150 for 3 hours. ONPs were then dehydrated sequentially in 10\%, 30\% and 50\% 151 methanol for 5 min each, followed by an air dried for 3 days. The samples 152 were dried again in a vacuum oven at room temperature overnight.

\section{Actin Staining}

154 After the cells were fixed in 4\% paraformaldehyde in PBS for $1 \mathrm{hr}$, they were 155 rinsed with PBS and permeabilised with 0.3\% Triton X-100 (PBST) for 15 min.

156 Three hundred and fifty $\mu$ of Texas-red-X phalloidin $(1: 150$, Invitrogen) was 157 then added to each well and left on the rocking plate for $1 \mathrm{hr}$. The cells were 
158 then rinsed with PBS after phalloidin was removed and cell nuclei were 159 counterstained with DAPI (1:200; BioLegend)

\section{Quantification of cell directionality}

161 Fluorescent images from actin staining were used for the quantification of cell 162 directionality. The images in red channel were converted to binary images 163 using the thresholding method and the orientation of cells on binary images 164 were then analysed by orientationJ distribution, an imageJ's plugin for 165 directional analysis. The distribution of orientation histogram was 166 automatically generated from each image.

\section{Calcein-AM staining}

168 Calcein-AM powder (Santa Cruz) was resuspended with anhydrous DMSO to 169 make a $2 \mathrm{mM}$ stock solution. Working solution was prepared immediately prior 170 to use by mixing Calcein-AM stock solution with the culture medium in 1:2000 171 dilutions. After the media was discarded, Calcein-AM working solution was 172 added to each well and incubated under $5 \% \mathrm{CO}_{2}$ at $37^{\circ} \mathrm{C}$ for $45 \mathrm{~min}$. The cells 173 were then washed with media and visualised under the fluorescent

174 microscope. Intracellular esterases in live cells hydrolyse a non-fluorescent 175 Calcein-AM to strongly fluorescent Calcein, which appeared green in cell 176 cytoplasm. Numbers of Calcein positive cells from at least 10 different fields 177 were used to calculate the estimated cell number on a $1 \mathrm{~cm}^{2}$ scaffold. 
180 deoxy-uridine (BrdU) labeling and detection kit I (Roche) was used to verify 181 the viability and proliferative capacity of ONPs on the scaffolds. Ten $\mu \mathrm{M}$ BrdU 182 was added to the culture medium and incubated for $48 \mathrm{hr}$ before they were 183 fixed with ethanol fixative, labelled with mouse anti-BrdU and anti-mouse 184 fluorescein. The concentration of reagents and antibodies were used 185 according to the manufacturer's instructions. Viable cells were then quantified 186 under fluorescent microscope.

\section{Immunolabelling and quantitation of fluorescence intensity}

After 14 days on the scaffolds, the ONPs were fixed with $4 \%$ paraformaldehyde in PBS for $1 \mathrm{hr}$ and permeabilised in $0.1 \%$ Triton $\mathrm{X}-100$ for

$19015 \mathrm{~min}$. Then, they were incubated for $1 \mathrm{hr}$ at room temperature in blocking 191 solution $(0.1 \%$ Triton $\mathrm{X}-100,5 \%$ normal donkey serum, $1 \%$ BSA in PBS) 192 before the primary antibodies were added. Double staining of $\beta$-tubulin III 193 (TUJ1) (1:100, mouse monoclonal, Covance) and NF200 (1:100, rabbit 194 polyclonal, Sigma), as well as Tau (1:50, rabbit polyclonal, Santa Cruz) and 195 MAP2 (1:200, mouse polyclonal, Abcam) were performed in this study. After 196 the cells were incubated with primary antibodies overnight at $4^{\circ} \mathrm{C}$, the 197 antibodies were rinsed off and secondary donkey anti-mouse or anti-rabbit 198 antibodies conjugated with Alexa Fluor 488 or Alexa Fluor 568 (1:250, 199 Invitrogen) were applied and incubated for $1 \mathrm{hr}$ at room temperature. For 200 nuclei labeling, the cells were counterstained with DAPI $(1: 200,4$,'6201 diamidino-2-phenylindole; BioLegend). 
Fluorescence intensity per cell was evaluated using ImageJ software

203 (NIH). All images were converted to black and white colour to avoid the bias.

204 Freehand selection was used to draw the area over each cells and the level of 205 fluorescence in a given region was measure as a mean grey value. This 206 number was then subtracted by the average background intensity of that 207 image.

\section{RNA extraction and gene expression analysis}

Total RNA was extracted using Trizol (Invitrogen) and Qiagen RNeasy

210 column combination protocol ${ }^{23} .1 \mathrm{ml}$ of Trizol was added to two $4 \mathrm{~cm}^{2}$

211 scaffolds and pipetted up and down to dissolve the fibres. After $5 \mathrm{~min}$ at room

212 temperature, $200 \mu$ of chloroform was added and mixed thoroughly. Each

213 tube was incubated for 2 min at room temperature, then spin down at 12,000

$214 \mathrm{~g}$ for $15 \mathrm{~min}$ at $4^{\circ} \mathrm{C}$. After the centrifugation, the transparent upper phase was

215 gently removed and transferred to a new tube. RNase-free $70 \%$ ethanol (500

$216 \mu \mathrm{l})$ was then added and mixed well using the pipette. The mixture was

217 transferred to the RNeasy mini spin column and preceded according the 218 manufacturer protocols.

219 QuantiTect Reverse Transcription Kit (Qiagen) was used to transcribe the 220 extracted RNA to cDNA. Then, to check the expression of neural markers, 221 real-time PCR was performed using Rotor-Gene SYBR Green (Qiagen). 222 Primer sequences of housekeeping and target genes (from 5' to 3') were as 223 follows: GAPDH, ATGGGGAAGGTGAAGGTCG and 224 TAAAAGCAGCCCTGGTGACC; NTNG1, TTGTGGATTGGAAAGGCTGC and 
225 GGTTGGAAGGGATTCAGGGA; NPR2, AACGCCATGCACCAGAAATT and

226 CATCTTCAGGCCAACAACCC; MMP13, TTGAGCTGGACTCATTGTCG and

227 TCTCGGAGCCTCTCAGTCAT.

\section{Statistical analysis}

229 All experiments were analysed using analysis of variance (one-way ANOVA)

230 followed by Bonferroni's post hoc comparison tests. Statistically significant

231 differences will be considered if the $p$-value is less than $0.05(p<0.05)$. All

232 values are shown as mean \pm standard deviation from at least 2 independent

233 experiments. All the graphs and statistic calculations were performed on

234 GraphPad Prism 6 (GraphPad Software, Inc., San Diego, CA, USA).

\section{RESULTS}

\section{Visualization and quantification of fibrous scaffolds}

238 After the fibres were fabricated, three independent electrospun scaffolds were

239 visualized and quantified. SEM micrograph and histogram of angle difference

240 demonstrated that most the fibres were aligned, with an average diameter of

$2410.93 \pm 0.1 \mu \mathrm{m}$ (Figure. $1 \mathrm{~A}$ and $\mathrm{B}$ ). Random fibres were also fabricated and

242 characterised to be used as a control in the experiments (Figure 1C). They

243 were spun with the same set up as in aligned-fibres but the collector was

244 rotated at the lowest speed. The distributions were almost equal in all angles

245 for random fibres (Figure 1D). 
247 The morphologies of hESC-derived ONPs grown under progenitor conditions 248 (OSCFM) for 3, 7 and 14 days on aligned PLLA fibres were investigated by 249 actin staining (Texas-red Phalloidin). Nuclei were counterstained with DAPI. 250 When cultured on tissue culture plastic for a few passages, ONPs were flat 251 and spread with an average size of $300 \mu \mathrm{m}$ (Figure 2A). On the contrary, a 252 distinct morphology was observed on 3D fibres, with cells being smaller with 253 an average size of $200 \mu \mathrm{m}$. After 3 days in culture, some of the ONPs were 254 spreading on the fibres (arrowhead) and elongated cells were noticed (arrow) 255 (Figure 2B). As for day 7 and 14, most of ONPs exhibited bipolar morphology $256(69 \pm 4 \%)$ and displayed a parallel, aligned pattern in some areas of the 257 scaffold (arrow) (Figure 2C). However, cells with polygonal morphology were 258 also presented (arrowhead). In some other areas on the scaffolds, 259 randomness of ONP cell arrangement still existed (Figure 2D). On the other 260 hand, ONP growing on the random PLLA fibres were spreading in all 261 directions and showed irregular morphology (Figure 2E).

262 SEM is another method that was used to confirm the findings from fluorescent 263 imaging. ONPs indeed extend along the fibre axes and the cells with different 264 orientation or irregular shape arise from the crossing fibres presence in the 265 scaffolds (Figure 2F,G). The micrographs also revealed that single ONP cells 266 attached to few fibres at a time.

267 Orientations of cell distributions were quantified using OrientationJ plugin from 268 ImageJ. The orientation of each pixel is evaluated and plotted as a histogram 269 against the orientations in degree. The histograms demonstrated that ONPs 

on 2D tissue culture plastic (Figure 2H) and PLLA random fibres (Figure 2J)

271 have broad distributions of orientation and several peaks were presented in 272 the histograms. While the cells on PLLA aligned fibres (Figure 2I) exhibited 273 one big peak, which indicated that most of the cells were arranged in a certain 274 degree.

\section{The effect of aligned PLLA fibres on ONPs viability and proliferation}

276 To check the number of viable ONP cells on the scaffolds, Calcein-AM 277 staining was carried out. After 14 days of culture, the number of viable cells 278 on neuralising 2D, control (OSCFM) PLLA and neuralising PLLA were all 279 significantly lower than those found on control (OSCFM) 2D (Figure 3A). The 280 lower numbers found on PLLA could be partially explained by the lower cell 281 attachment expected on PLLA fibres, as no bioactive sites are presented and 282 they were not treated with any chemicals or biological compounds that would 283 enhance cell attachment.. A decreased cell number under neuralising 284 condition could also infers that ONPs are differentiating into non-proliferating 285 neurons ${ }^{24}$. Therefore, the population of cells with dividing capability is lower. 286 It is noted that the differences between control and neuralising conditions on $2872 \mathrm{D}$ were larger than those on PLLA.

288 The proliferation of ONPs was also investigated using a BrdU incorporation 289 assay (figure 3B). A significant reduction of cell proliferation, triggered by 290 culturing cells on PLLA was noticed after 3 days, regardless of the culture 291 conditions. The trend was maintained after 14 days, although the differences 292 were not large enough to remain significant. . The reduction of the percentage 293 of BrdU positive cells in neuralising conditions was, however, more obvious. 


\section{The effect of aligned PLLA fibres on ONPs differentiation}

295 We investigated the differentiation behaviour of ONPs on PLLA electrospun

296 fibres in both conditions (progenitor and neuralising) using immunostaining of 297 the early neuronal marker $\beta$-tubulin III (TUJ1), and the mature neural marker 298 neurofilament-200 (NF200). After 14 days in culture, the expressions of TUJ1 299 and NF200 were enhanced when the cells were grown in the neuralising 300 condition or on aligned fibres (noted that cell number per area on the fibres 301 are lower than those on 2D) (Figure 4A). Mean Fluorescent intensity per cell 302 and number of positive cells for both markers were increased even without 303 any neurotrophins supplement (Figure 4B). On 2D, the intensities of both 304 markers were relatively the same in control and neuralising conditions but 305 numbers of positive cells were higher in the neuralising one. Similar trend was 306 observed on random fibres with a slight increase of fluorescence intensity. 307 The significant difference was detected only on TUJ1 between control 2D and 308 neuralising random fibres.

309 Expression of both neuronal markers studied was enhanced on ONPs seeded 310 on aligned fibres under both culture conditions. There was a clear, statistically 311 significant shift of labelling intensities $(p<0.0001)$. This indicated that the 312 alignment of fibres alone could effectively drive the differentiation of ONPs.

313 Neuralising condition increased the neuronal marker expression on aligned 314 fibres even further and the intensity of both TUJ1 and NF200 were 315 significantly increased comparing to control condition. .

316 Several molecular markers known to be highly expressed in differentiated 317 ONPs were also explored. Matrix metalloproteinase (MMP13), natriuretic 
peptide receptor2 (NPR2) and Netrin-G1 (NTNG1) ${ }^{4}$, were used as

319 differentiation marker genes and analysed by qRT-PCR (Figure 4C). These

320 genes are upregulated in a specific manner during SGN development.

321 MMP13, also known as collagenase-3, is a zinc-dependent proteolytic

322 enzyme involved in the remodeling of ECM and is enriched at late

323 developmental stage of SGN (Lu et al., 2011; NCBI, 2016a). NPR2 encodes

324 an integral membrane receptor for natriuretic peptides, which is required for

325 axon bifurcation in SGN and auditory circuit assembly ${ }^{25,27,28}$. NPR2 is highly

326 expressed at very early stage, then dropped in the middle and finally raised

327 again in the late developmental stage of SGN. NTNG1 encodes a

328 preproprotein that can be further processed into a secreted protein, which is

329 responsible for axon growth and guidance. It is highly expressed throughout

330 the SGN development but the level is slightly higher in the early stage ${ }^{25,29}$.

331 All three genes were upregulated when ONPs were cultured in neuralising 332 medium and on the aligned scaffolds. The increment ranged from 1 to 6 folds.

333 The upreguration of MMP13 and NPR2 on aligned fibres were even higher 334 than those on 2D neuralising conditions. On the contrary, the induction of 335 NTNG1 was maximised on 2D neuralising condition. However, due to wide 336 deviations, none of them are considered as significantly different. From the 337 pattern of gene expression that were observed, it can be inferred that after 14 338 days of differentiation, ONPs on aligned fibres are in a late developmental 339 stage. 


\section{The effect of aligned PLLA fibres on ONPs polarization}

341 To verify the polarisation of differentiated cells, ONPs were fixed and co-

342 stained with anti-TAU (axonal marker) and anti-MAP2 (dendritic marker)

343 antibodies after 14 days under different culture conditions. On TC plastic,

344 ONPs under progenitor condition rarely expressed TAU and MAP2 but a slight

345 increase of TAU expression was observed and cells with positive MAP2 were

346 identified under neuralising condition (Figure. 5A). On align fibres, ONPs in

347 both progenitor and neuralising conditions were positive for both neuronal

348 polarity markers. Some ONPs also showed specific localization of MAP2 in

349 the soma and TAU in both soma and axon indicating a polarization of those

350 cells (arrow). However, the majority of the cell population did not yet specify

351 their neuronal polarity. The number of positive cells was then quantified and

352 the results revealed that neuralising 2D, control PLLA and neuralising PLLA

353 can increase the percentage of TAU and MAP2 positive cells by 2 to 8 folds

354 when comparing to Control 2D group (Figure 5B). The percentages of those 3

355 conditions were comparable but the most prominent one was observed on

356 neuralising PLLA.

\section{Discussion}

358 Not only limited to mature cells, the correlation between substrate properties

359 or microenvironment and stem cell fate has been widely investigated. Stem

360 cell differentiation is one of the main interests in tissue engineering and

361 regeneration, as in many cases the desired cell type is required prior to

362 transplantation for the safety and efficiency of the treatment ${ }^{30}$. It is

363 increasingly clear that the native microenvironment that supports stem cells or 
364 'stem cell niche' plays a crucial role in the control of stem cell behaviour and

365 fate decision ${ }^{3132}$. Thus, a considerable amount of work is currently being 366 performed employing this view that allows taking control over stem cells and 367 directing them to the desired behaviour.

368 Numerous studies have explored the use of electrospun fibres as tissue 369 engineering scaffolds for the repair of both central and peripheral nervous 370 system injuries ${ }^{33,34}$. Aligned electrospun fibres have also been used by many 371 research groups. Its advantages in neurite guiding and extension has been 372 reported in vitro ${ }^{35-37}$ and the success repair were also demonstrated in vivo 373 38,39. However, those studies were performed with the cells that are not 374 directly relevant to the auditory system and tissue engineering for SGN 375 damage or degeneration has never been tried. Here, we investigated the 376 response of ONPs, a promising cell type that could be used for SGN 377 regeneration, to the aligned fibrous tissue-engineered substrate (PLLA).

378 There are many parameters that affect electrospinning process and fibre 379 morphology. They are needed to be optimised in order to achieve the ideal 380 scaffolds for each work ${ }^{40}$. So, in order to obtain an aligned fibre mesh with 381 the diameter of $\sim 1 \mu \mathrm{m}$, which is expected to be the compromise fibre size for 382 neuronal guidance, proliferation and differentiation ${ }^{41,42}$, electrospinning 383 parameters were manipulated. The most suitable parameters obtained were 384 used to produce aligned and random electrospun fibres. The visualisation and 385 quantification of SEM images showed that we can successfully obtain both 386 fibre types. 
387 One of the very first ONP features that we monitored when grown on PLLA 388 fibres was cell morphology. The dissimilarity of ONPs morphology on 2D and $3893 \mathrm{D}$ matrices are to be expected as the cells that were cultured on 2D tissue 390 culture plastic are flat and can only spread in horizontal plane, while on 3D, 391 which is considered to be closer to physiological conditions, cells can spread 392 in all directions ${ }^{43}$. The parallel cell pattern is a promising result indicating that 393 alignment of the fibres can act as a guidance cues for ONPs. Calcein-AM 394 staining and BrdU assay also demonstrated that the numbers of viable, 395 attached cells and the percentage of cell proliferation were lower on the fibres 396 comparing to 2D culture plastic. These findings are coherent with the idea 397 cells are differentiating, reducing or stopping proliferation altogether ${ }^{24}$.

398 In previous studies of the role of scaffolds on neural differentiation, 399 experiments have been performed under neural-inducing conditions such the 400 presence of neurotrophins. However, the effect of the external scaffold on the 401 progenitors is rarely described ${ }^{44-46}$. From the expression of neuronal markers 402 in protein and RNA level, we clearly showed that ONPs were sensitive to the 403 change of physical environment (aligned fibres) and were able to differentiate 404 even in the normal expansion medium. Under neuralising condition, ONP 405 differentiation was increased even further. This indicated that there is a 406 synergistic effect between physical (fibre alignment) and chemical cues 407 (neuralising agents).

408 In order for neural cells to function after they are differentiated, polarization 409 and/or establishment of axons and dendrites are required ${ }^{47}$. Beside from 410 intrinsic factors, extracellular signal also controls polarity specification as the 
411 neural cells are physiologically surrounded by extracellular matrix. Various

412 axonal guidance and repellent molecules have been identified to effectively

413 control the asymmetric growth of neurites and leads to axon or dendrite

414 formation ${ }^{48,49}$. Some growth factors such as NT3 and BDNF that were used in

415 our differentiation medium also help enhancing neuronal polarization ${ }^{50,51}$. As

416 we previously found that ONPs are differentiated on the aligned fibres and lots

417 of them are positive for mature neuronal marker (NF200), the establishment of

418 neural polarity is another aspect to confirm the transition to a mature neuronal

419 phenotype. Culturing of ONPs on aligned scaffolds and under neuralising

420 condition increased the expression of neuronal polarity markers (TAU and

421 MAP2) but the polarities of those positive cells were not yet to be specified.

\section{FIGURES}


A.

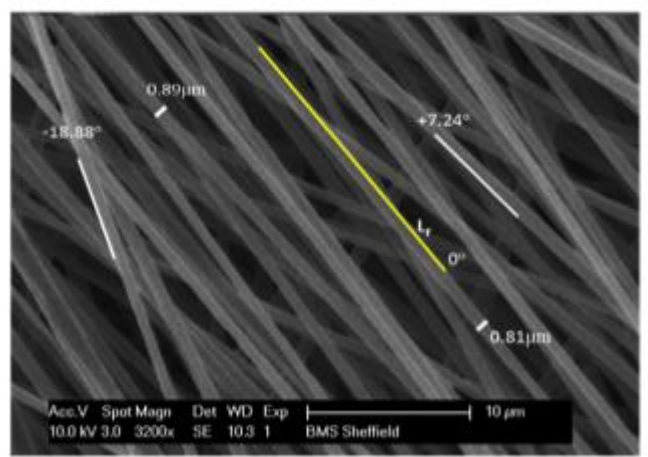

C.

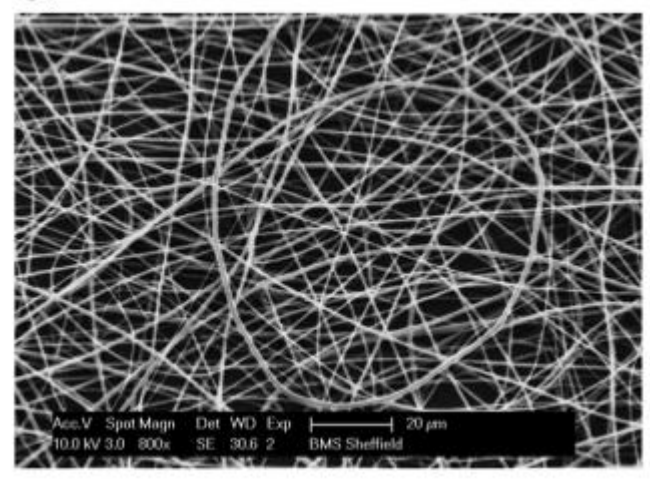

B.

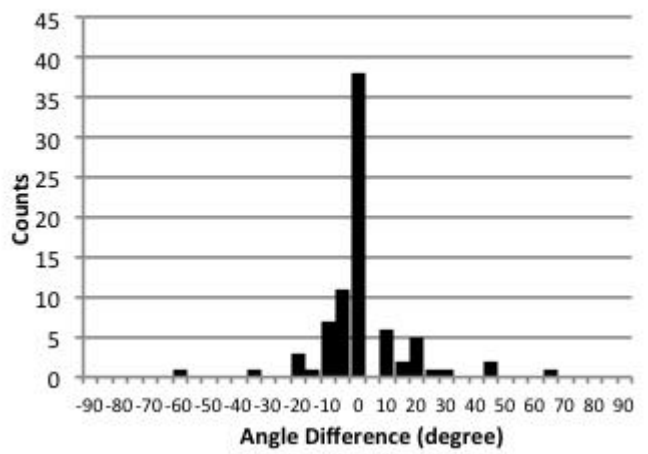

D.

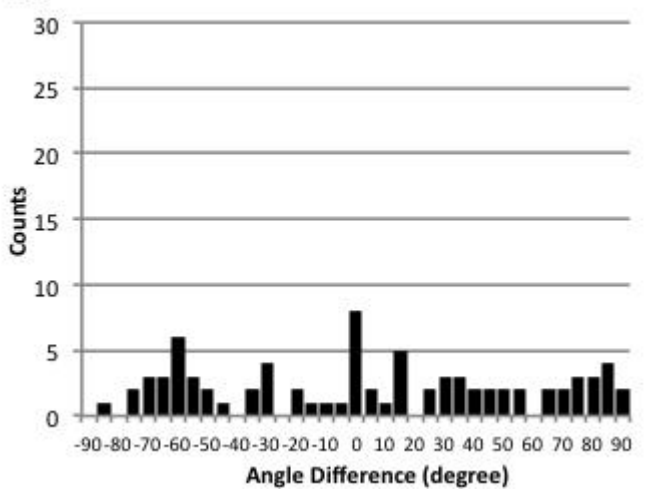

426 Figure 1. Electrospun fibres from optimized electrospinning parameters. (A) SEM 427 image of PLLA aligned fibres and alignment analysis (scale bar $=10 \mu \mathrm{m}$ ). (B) 428 Histogram of angle difference showing that most of the fibres are parallel to the 429 reference line. (C) SEM image of random fibres collected with the lowest collector 430 rotation speed (scale bar $=20 \mu \mathrm{m}$ ). (D) Histogram of angle difference on random 431 fibres. 
437

438

439
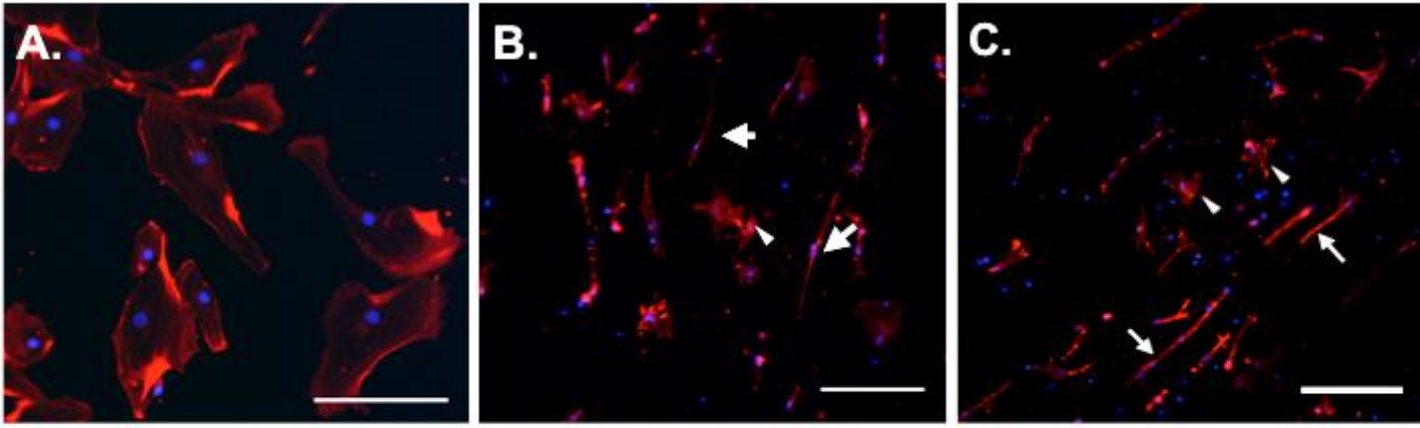

440
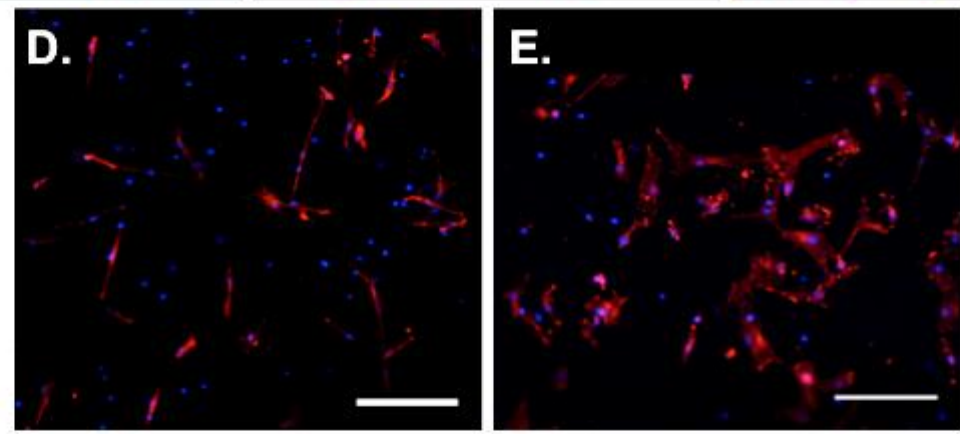

443
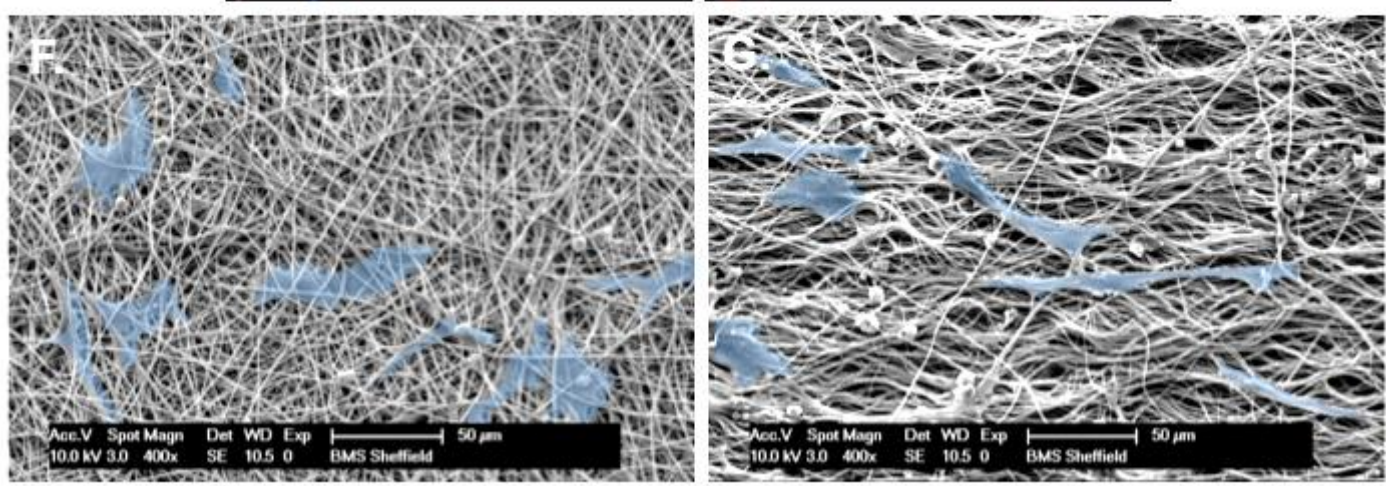

446

447

H.
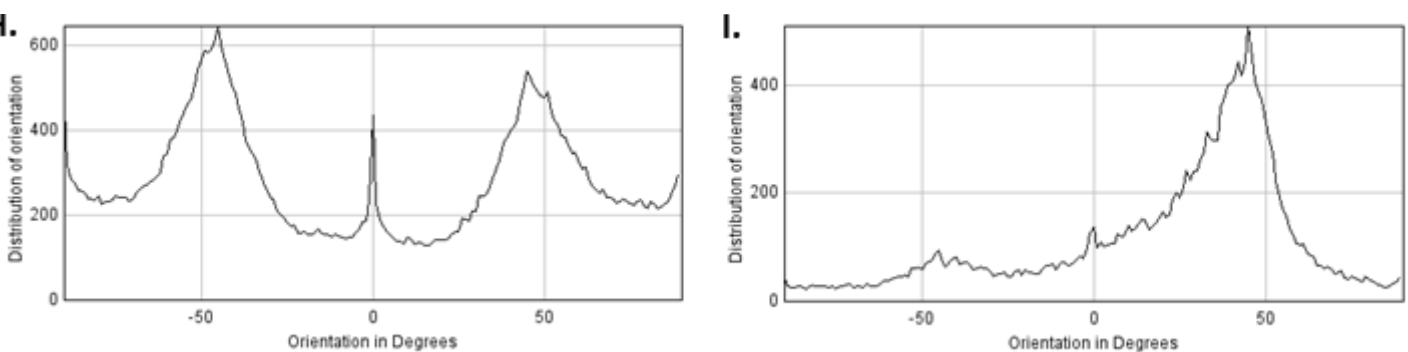

449

450

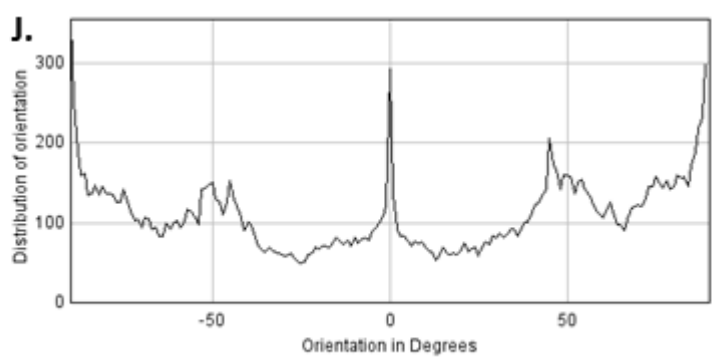

452 Figure 2. Actin staining showing ONP cell morphology on (A) tissue culture

453 plastic (B) PLLA aligned fibres after 3 days in culture (C,D) PLLA aligned

454 fibres after 14 days in culture (E) PLLA random fibres after 14 days in culture. 
455 Scale bar=200 $\mu \mathrm{m}$. Scanning electron micrographs of the cells were shown on

456 (F) random and (G) aligned PLLA fibres. The cells were masked in blue for 457 the visibility. Scale bar $=50 \mu \mathrm{m}$. Distribution of cell orientation on tissue culture 458 plastic, PLLA aligned and random fibres were measured and shown as 459 histograms on $(\mathrm{H}),(\mathrm{I})$ and $(\mathrm{J})$ respectively.

A.

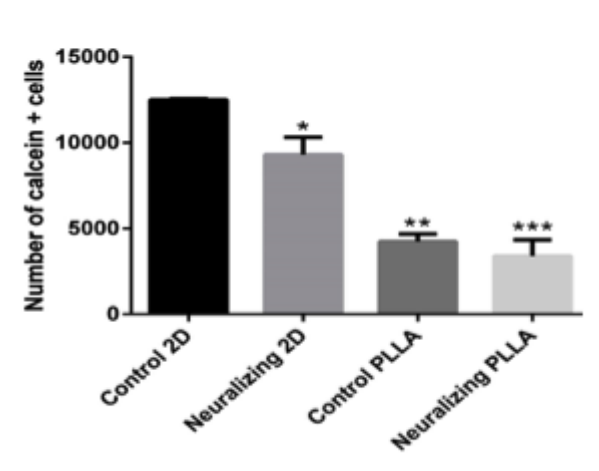

B.

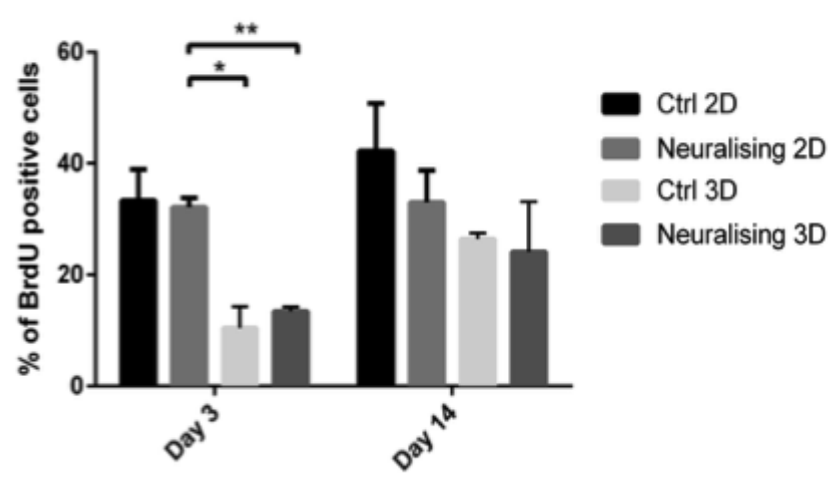

461 Figure 3. Viability and proliferation of ONPs on PLLA fibres. (A) Number of viable 462 ONPs on $1 \times 1 \mathrm{~cm}^{2}$ PLLA aligned fibres after 14 days in culture. Data is mean $\pm S D$. (B) 463 ONPs proliferation after 3 and 14 days culture on PLLA aligned fibres. ${ }^{*} p<0.05$, $464{ }^{* *} p<0.01$ 
A.
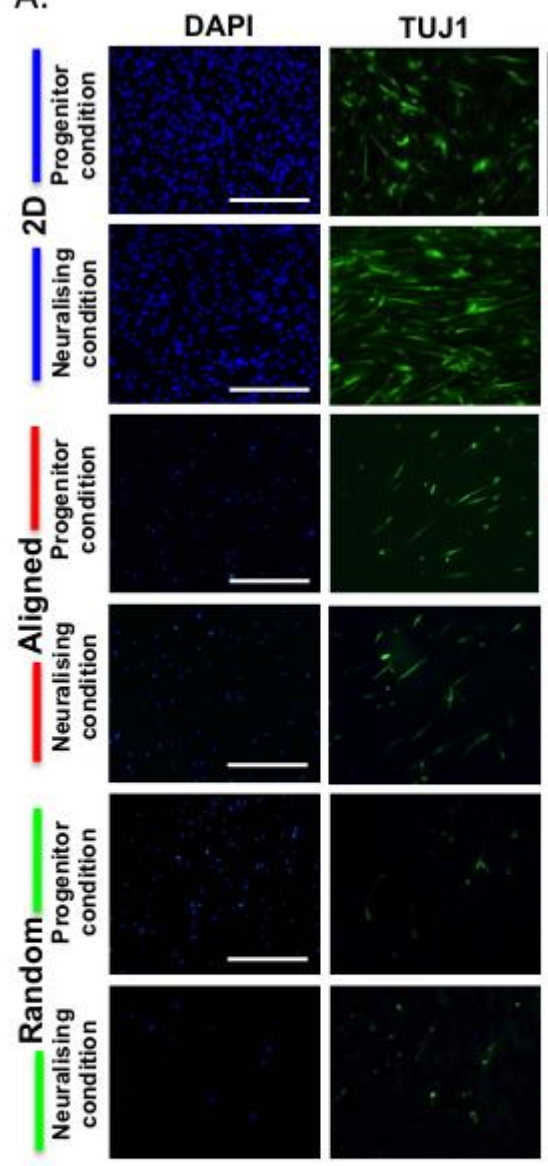

MMP13

D.

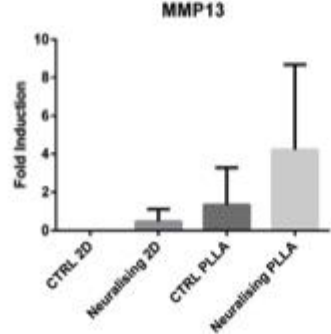

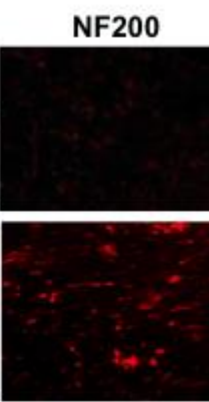
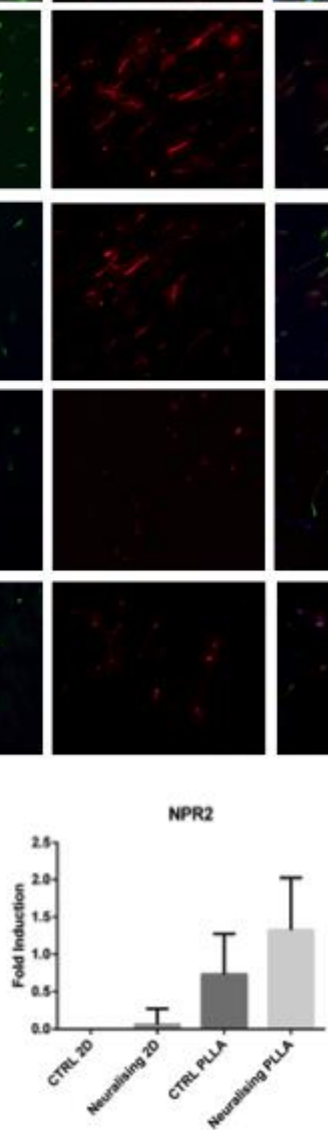

Merge
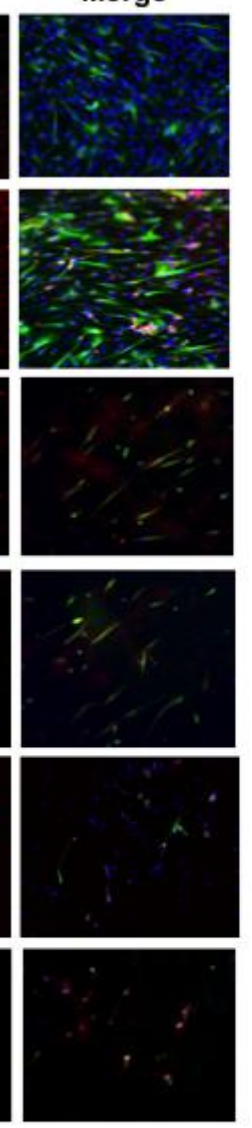

B.

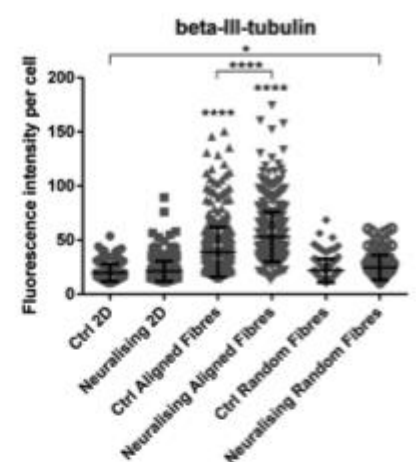

C.
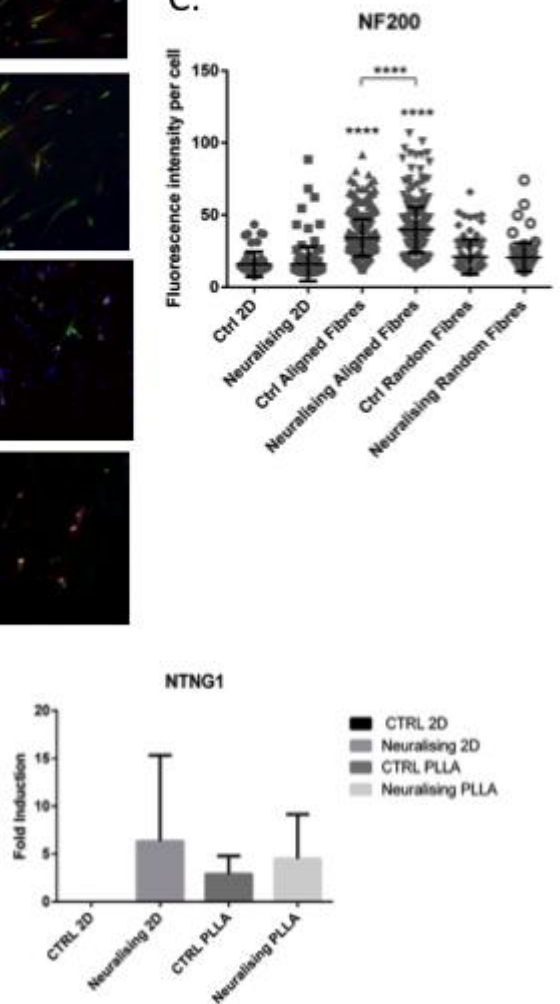

467 Figure 4. The differentiation of ONPs on PLLA fibres. (A) Immunostaining of ONPs 468 on 2D tissue culture plastic and 3D aligned PLLA fibres for neuronal markers, TUJ1 469 or B-tubulinlll (green) and NF200 (red). Scale bar=400 $\mu \mathrm{m}$. (B) Mean fluorescent 470 intensity per cell of B-tubulinIII and (C) NF200 on different culture materials. ${ }^{*} \mathrm{p}<0.05$, $471^{* * * *} p<0.0001$ (D) The effect of aligned substrates on the expression of MMP13, 472 NPR2 and NTNG1 in ONPs. Data is mean \pm SD. 
A.
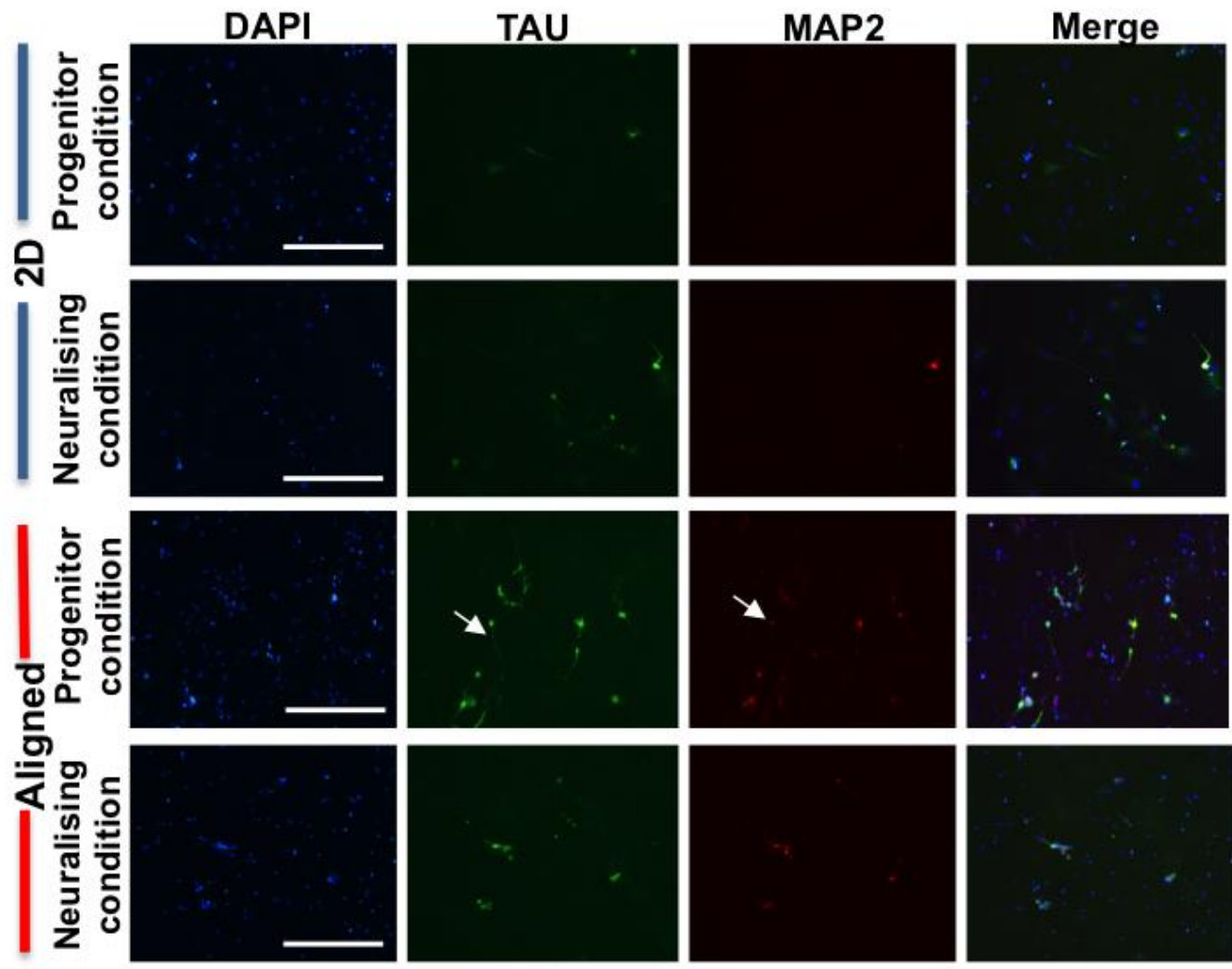

B.

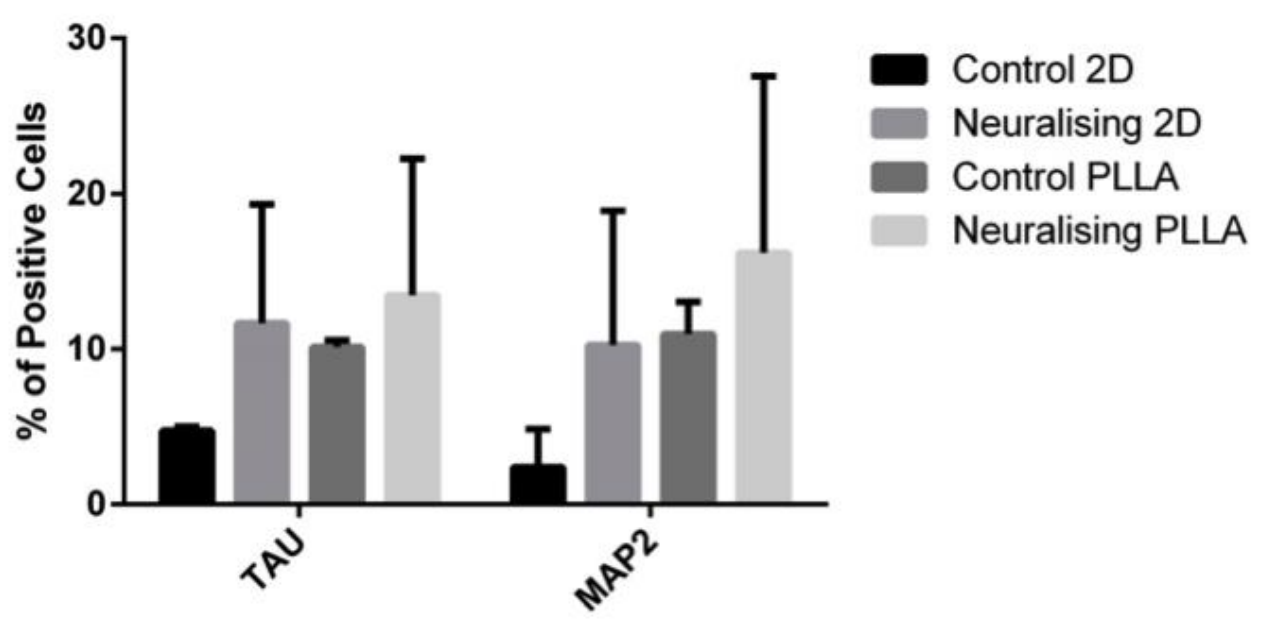

474 Figure 5. ONP polarization on PLLA aligned fibres. (A) Immunolabelling of neural 475 polarity markers, TAU (green) and MAP2 (red) on ONPs. Scale bar=400 $\mu \mathrm{m}$. (B) 476 Percentage of TAU and MAP2 positive ONPs when cultured on different substrates 477 for 14 days. Data shown are mean \pm SD. 


\section{CONCLUSIONS AND FUTURE WORKS}

479 In summary, we have demonstrated that otic neural progenitors (ONPs) can 480 attach, survive and be able to differentiate on aligned PLLA fibres. The 481 physical cue (alignment of fibres) can help enhancing the differentiation of 482 ONPs even in the absence of neuralising agents and guiding neurite 483 extension along the alignment axis. In addition, it also influences changes on 484 ONP morphology and the specification of neural polarity. This aligned PLLA 485 scaffolds can also be further manipulated to facilitate the transplantation. A 486 conduit could be made by rolling the PLLA electrospun sheet and seed it with 487 ONPs and glial cells. These applications could be highly useful to develop 488 new strategies to repair the auditory nerve.

\section{ACKNOWLEDGEMENT}

490 The authors would like to thank Dr. Priya Vishwanathan and Rivolta's lab 491 members for their kind help in teaching new lab techniques. This work was 492 supported by the Royal Thai Government and partially supported by MRC 493 award to Prof. Marcelo N. Rivolta.

\section{CONTRIBUTIONS}

495 All authors participate in the designed and interpretation of the studies. KN 496 performed the experiments, analysed the data and wrote the manuscript. 497 MNR and GB contributed to the revision of the manuscript. 


\section{COMPETETING INTERESTS}

500 The authors declare no conflict of interest.

\section{REFERENCES}

503 1. Groves, A. K., Zhang, K. D. \& Fekete, D. M. The genetics of hair cell 504 development and regeneration. Annu. Rev. Neurosci. 36, 361-81 (2013).

505 2. Hu, Z. et al. Survival and neural differentiation of adult neural stem cells 506 transplanted into the mature inner ear. Exp. Cell Res. 302, 40-47 (2005).

507 3. Incesulu, A. \& Nadol, J. Correlation of acoustic threshold measures and spiral ganglion cell survival in severe to profound sensorineural hearing loss: Implications for cochlear implantation. Ann. Otol. Rhinol. Laryngol. 107, 906911 (1998).

511 4. Chen, W. et al. Restoration of auditory evoked responses by human ES-cellderived otic progenitors. Nature 490, 278-282 (2012).

513 5. Lang, H., Schulte, B. A. \& Schmiedt, R. A. Ouabain induces apoptotic cell

514 death in type I spiral ganglion neurons, but not type II neurons. JARO - J.

515 Assoc. Res. Otolaryngol. 6, 63-74 (2005).

516 6. Burdick, J. A. \& Mauck, R. L. Biomaterials for Tissue Engineering Applications. 517 (Springer-Verlag Wien, 2011).

518 7. Discher, D. E., Mooney, D. J. \& Zandstra, P. W. Growth factors, matrices, and 
519 forces combine and control stem cells. Science 324, 1673-7 (2009).

520 8. Shibata, S. B. \& Raphael, Y. Future approaches for inner ear protection and repair. Journal of Communication Disorders 43, 295-310 (2010).

9. Clarke, J. C. et al. Micropatterned methacrylate polymers direct spiral ganglion neurite and Schwann cell growth. Hear. Res. 278, 96-105 (2011).

10. Mattotti, M., Micholt, L., Braeken, D. \& Kovačić, D. Characterization of spiral ganglion neurons cultured on silicon micro-pillar substrates for new auditory neuro-electronic interfaces. J. Neural Eng. 12, 26001 (2015).

527 11. Reich, U. et al. Differential fine-tuning of cochlear implant material-cell 528 interactions by femtosecond laser microstructuring. J. Biomed. Mater. Res. Part B Appl. Biomater. 87, 146-153 (2008).

12. Stöver, T. \& Lenarz, T. [Biomaterials in cochlear implants]. Laryngorhinootologie. 88 Suppl 1, S12-S31 (2009).

13. Evans, A. R. et al. Laminin and fibronectin modulate inner ear spiral ganglion neurite outgrowth in an in vitro alternate choice assay. Dev. Neurobiol. 67, $1721-1730$ (2007).

14. Kikkawa, Y. S. et al. Growth factor-eluting cochlear implant electrode: impact on residual auditory function, insertional trauma, and fibrosis. J. Transl. Med. 12, 280 (2014).

15. Paasche, G. et al. Technical report: modification of a cochlear implant electrode for drug delivery to the inner ear. Otol. Neurotol. 24, 222-7 (2003). 
540 16. Pettingill, L. N., Richardson, R. T., Wise, A. K., O'Leary, S. J. \& Shepherd, R.

$541 \quad$ K. Neurotrophic factors and neural prostheses: Potential clinical applications 542 based upon findings in the auditory system. IEEE Transactions on Biomedical Engineering 54, 1138-1148 (2007).

544 17. Ryan, A. F., Wittig, J., Evans, A., Dazert, S. \& Mullen, L. Environmental 545 micropatterning for the study of spiral ganglion neurite guidance. Audiol. Neurotol. 11, 134-143 (2005).

18. Stover, T. et al. Development of a drug delivery device: using the femtosecond laser to modify cochlear implant electrodes. Cochlear Implant. Int 8, 38-52 (2007).

19. Xie, J. et al. Neurotrophins differentially stimulate the growth of cochlear neurites on collagen surfaces and in gels. NEURAL Regen. Res. 8, (2013).

20. Schwieger, J. et al. Neuronal survival, morphology and outgrowth of spiral ganglion neurons using a defined growth factor combination. PLoS One 10, (2015).

21. Santi, P. A. \& Johnson, S. B. Decellularized ear tissues as scaffolds for stem cell differentiation. JARO - J. Assoc. Res. Otolaryngol. 14, 3-15 (2013).

22. Chen, W. et al. Human fetal auditory stem cells can be expanded in vitro and differentiate into functional auditory neurons and hair cell-like cells. Stem Cells 27, 1196-1204 (2009). (2008). 
563 24. Purves, D. et al. Neuroscience. (Sinauer Associate, Inc., 2008). doi:9780878937257

25. Lu, C. C., Appler, J. M., Houseman, E. A. \& Goodrich, L. V. Developmental Profiling of Spiral Ganglion Neurons Reveals Insights into Auditory Circuit Assembly. J. Neurosci. 31, 10903-10918 (2011).

26. NCBI. MMP13 matrix metallopeptidase 13 [ Homo sapiens (human) ]. (2016). at <http://www.ncbi.nlm.nih.gov/gene/4322>

27. Lu, C. C. et al. Mutation of Npr2 Leads to Blurred Tonotopic Organization of Central Auditory Circuits in Mice. PLoS Genet. 10, (2014).

28. NCBI. NPR2 natriuretic peptide receptor 2 [ Homo sapiens (human) ]. (2016). at <http://www.ncbi.nlm.nih.gov/gene/4882>

29. NCBI. NTNG1 netrin G1 [ Homo sapiens (human) ]. (2016). at $<$ http://www.ncbi.nlm.nih.gov/gene/22854>

30. Guillot, P. V, Cui, W., Fisk, N. M. \& Polak, D. J. Stem cell differentiation and expansion for clinical applications of tissue engineering. J. Cell. Mol. Med. 11, 935-44 (2007).

579 31. Schofield, R. The relationship between the spleen colony-forming cell and the haemopoietic stem cell. Blood Cells 4, 7-25 (1978).

581 32. Li, L. \& Xie, T. Stem cell niche: structure and function. Annu. Rev. Cell Dev. Biol. 21, 605-631 (2005). 
medicine. Adv. Healthc. Mater. 1, 10-25 (2012).

585

586

587

588

589

590

591

592

593

594

595

596

597

598

599

600

601

602

603

604

605

34. Xie, J., MacEwan, M. R., Schwartz, A. G. \& Xia, Y. Electrospun nanofibers for neural tissue engineering. Nanoscale 2, 35-44 (2010).

35. Schnell, E. et al. Guidance of glial cell migration and axonal growth on

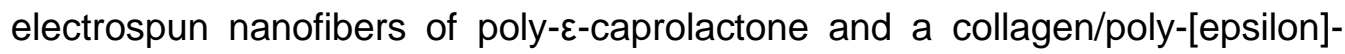
caprolactone blend. Biomaterials 28, 3012-3025 (2007).

36. Shen, Y. et al. Guidance of olfactory ensheathing cell growth and migration on electrospun silk fibroin scaffolds. Cell Transplant. 19, 147-157 (2010).

37. Xie, J. et al. The differentiation of embryonic stem cells seeded on electrospun nanofibers into neural lineages. Biomaterials 30, 354-362 (2009).

38. Hurtado, A. et al. Robust CNS regeneration after complete spinal cord transection using aligned poly-I-lactic acid microfibers. Biomaterials 32, 6068$6079(2011)$.

39. Kim, Y. tae, Haftel, V. K., Kumar, S. \& Bellamkonda, R. V. The role of aligned polymer fiber-based constructs in the bridging of long peripheral nerve gaps. Biomaterials 29, 3117-3127 (2008).

40. Bhardwaj, N. \& Kundu, S. C. Electrospinning: A fascinating fiber fabrication technique. Biotechnology Advances 28, 325-347 (2010).

41. Binder, C., Milleret, V., Hall, H., Eberli, D. \& Lühmann, T. Influence of micro and submicro poly(lactic-glycolic acid) fibers on sensory neural cell locomotion and neurite growth. J. Biomed. Mater. Res. - Part B Appl. Biomater. 101, $1200-1208$ (2013). 
606 42. Christopherson, G. T., Song, H. \& Mao, H. Q. The influence of fiber diameter of electrospun substrates on neural stem cell differentiation and proliferation. Biomaterials 30, 556-564 (2009).

43. Baker, B. M. \& Chen, C. S. Deconstructing the third dimension - how 3D culture microenvironments alter cellular cues. J. Cell Sci. 125, 3015-3024 (2012).

44. Gerardo-Nava, J. et al. Human neural cell interactions with orientated electrospun nanofibers in vitro. Nanomedicine (Lond). 4, 11-30 (2009). nanofiber-guided cell alignment on the preferential differentiation of neural stem cells. Biomaterials 31, 9031-9039 (2010).

46. Liu, X. et al. Guidance of neurite outgrowth on aligned electrospun polypyrrole/ poly(styrene-beta-isobutylene-beta-styrene) fiber platforms. J. Biomed. Mater. Res. - Part A 94, 1004-1011 (2010).

47. Arimura, N. \& Kaibuchi, K. Neuronal polarity: from extracellular signals to intracellular mechanisms. Nat. Rev. Neurosci. 8, 194-205 (2007).

48. Adler, C. E., Fetter, R. D. \& Bargmann, C. I. UNC-6/Netrin induces neuronal asymmetry and defines the site of axon formation. Nat. Neurosci. 9, 511-518

49. Whitford, K. L., Dijkhuizen, P., Polleux, F. \& Ghosh, A. Molecular control of cortical dendrite development. Annu. Rev. Neurosci. 25, 127-49 (2002).

627 50. Da Silva, J. S., Hasegawa, T., Miyagi, T., Dotti, C. G. \& Abad-Rodriguez, J. 
Asymmetric membrane ganglioside sialidase activity specifies axonal fate. Nat Neurosci 8, 606-615 (2005).

630 51. Huang, E. J. \& Reichardt, L. F. Trk Receptors: Roles in Neuronal Signal Transduction. Annu. Rev. Biochem. 72, 609-642 (2003). 